Abstract PS-095a Table 1

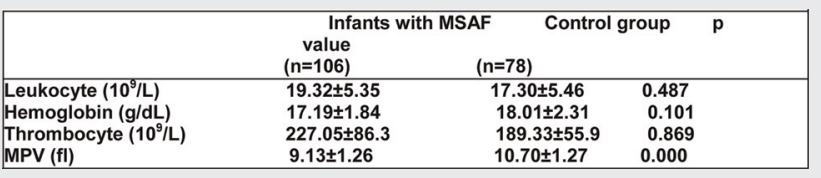

Background and aims To investigate the association between serum mean platelet volume (MPV) levels and meconium stained amniotic fluid (MSAF).

Methods MPV, serum reactive protein and haemoglobin levels, leukocyte and thrombocyte counts were measured in 106 infants with MSAF and 78 healthy control infants for comparison. Demographic factors including gestational age, birth weight, gender, delivery mode, parity, maternal pregnancy diseases, intrauterine infections, maternal smoking and severity of meconium aspiration syndrome were recorded.

Results The mean values of MPV in infants with MSAF were significantly lower than in the control group statistically $(p<$ 0.001 ). There was no statistically significant difference in haemoglobin levels, and leukocyte and thrombocyte counts in the study group compared to the control group $(\mathrm{p}>0.05)$. There was no statistically significant difference in MPV levels in the infants with meconium aspiration syndrome (MAS) compared to the study group ( $\mathrm{p}>0.05$ ). A $9.90 \mathrm{fl}$ [area under the curve (AUC: $0.788)$ ] optimal cutoff value of MPV with a sensitivity of $78.1 \%$ and specificity of $74.3 \%$ was determined in infants with MSAF. Conclusions Our data suggest that the MPV levels were significantly lower in infants with MSAF than that in healthy infants. This might be associated with hypoxic process. However, the MPV levels were statistically similar between MSAF and MAS, leading to a failure of detecting patients with or without severe disease.

\section{PS-096 FEASIBILITY AND SAFETY OF DUCTAL CLOSURE USING AMPLATZER PDA OCCLUDER ADO IIAS IN NEWBORN LESS THAN 2500 GRAMS}

P Morville, A Akhavi, A Charbonneau. NICU Pediatric Cardiology, American Memorial Hospital, Reims, France

\subsection{6/archdischild-2014-307384.391}

Objective To evaluate feasibility and safety of patent ductus arteriosus (PDA) occlusion in very low birth weight newborns $<2500 \mathrm{~g}$ when surgery was indicated by PDA occluder under X ray and ultrasounds

Methods After ultrasound evaluation and Ibuprofen treatment when required, significant haemodynamic PDA were defined by high flow in pulmonary artery branch $>45 \mathrm{~cm} / \mathrm{sec}$, absence or reverse flow in middle cerebral artery, renal and mesenteric arteries, left to right shunt, and DA diameter. Interventional catheterism was proposed as first option. Babies were anaesthetised with ketamine and sufenta. Femoral vein access by 4F Desilets. A catheter was positionned in descending thoracic aorta and the PDA II AS occluder positionned under horizontal $\mathrm{X}$ ray and ultrasound. Temperature was kept between 35.5 and $36^{\circ} \mathrm{C}$ along the procedure.

Results Twelve newborns had a PDA closure. Mean weight was $1740 \mathrm{~g}$ (850-2480). The series started at the highest weight. Mean diameter of the duct was $3,3 \mathrm{~mm}(2,2-4)$ and length 6 (2-11). PDA ADO IISA used were $3 \times 4(2) 4 \times 2(1), 4 \times 4(4)$,
$4 \times 6(2), 5 \times 2(2)$ and $5 \times 6(1)$. Two were repositioned. Mean duration of the entire procedure was $32 \mathrm{~min}(10-90)$. X ray exposure was $12 \mathrm{~min}$ (3-24) and dose 1,5 grey/cm2 (0.2-4). Two transitional paraprothetic shunts closed spontaneously after few days. One premature infant already in renal failure, died ten days later despite ductal closure. In the others the follow up showed improvement in cerebral and pulmonary blood flows without any haemodynamic problems such as observed after surgical procedures.

Conclusion This preliminary series showed feasibility and safety when combining X-ray and US to close a PDA with PDA ADO IIAS.

\section{PS-097 ADULT SURVIVORS OF FETAL ANAEMIA HAVE INCREASED CARDIOVASCULAR RISK: FINDINGS FROM A COHORT STUDY OF INTRAUTERINE TRANSFUSION RECIPIENTS}

${ }^{1} \mathrm{AH}$ Wallace, ${ }^{2} \mathrm{SR}$ Dalziel, ${ }^{3} \mathrm{BR}$ Cowan, ${ }^{3} \mathrm{AA}$ Young, ${ }^{4} \mathrm{KL}$ Thornburg, ${ }^{1} \mathrm{JE}$ Harding. 'Liggins Institute, University of Auckland, Auckland, New Zealand; ${ }^{2}$ Children's Emergency Department, Starship Children's Hospital, Auckland, New Zealand; ${ }^{3}$ Department of Anatomy with Radiology, University of Auckland, Auckland, New Zealand; ${ }^{4}$ Heart Research Center, Oregon Health and Sciences University, Portland, USA

\subsection{6/archdischild-2014-307384.392}

Background In sheep, intrauterine anaemia causes increased susceptibility to ischaemic injury in adulthood, but effects on humans are unknown. The aim of this study was to investigate cardiovascular outcome in adults who received intrauterine transfusion for fetal anaemia due to rhesus disease.

Methods Participants were adults who received intrauterine transfusion and their unaffected sibling (s). Assessments included anthropometry, blood pressure, lipids, heart rate variability and cardiac MRI. Analysis was by multiple regression adjusted for age, sex, and birth weight z-score, with data as adjusted means \pm SD (95\% CI).

Results Compared to unaffected siblings, affected participants had smaller ventricular volumes, for example end diastolic volume indexed to body surface area (EDV/BSA); reduced high density lipoprotein (HDL); increased heart rate variability low frequency/high frequency (LF/HF) ratio indicating augmented sympathetic tone; and lower myocardial blood flow (MBF) at rest and with cold pressor stress, but not during adenosine infusion, indicating impaired endothelial function.

Conclusions Endothelial dysfunction in adults exposed to fetal anaemia may be mediated by augmented sympathetic tone. Taken together with smaller ventricular volumes and reduced HDL, this study provides the first evidence in humans that fetal anaemia may be associated with increased cardiovascular risk in adulthood.

\begin{tabular}{llll} 
Abstract PS-097 Table 1 & & \\
\hline & $\begin{array}{l}\text { Affected } \\
(\mathrm{n}=95)\end{array}$ & $\begin{array}{l}\text { Unaffected } \\
(\mathrm{n}=92)\end{array}$ & p-value \\
\hline Age (years) & $33.7 \pm 9.3$ & $40.1 \pm 10.9$ & $<0.001$ \\
EDV/BSA (ml/m²) & $79.3(76.9-81.7)$ & $85.4(83.0-87.9)$ & 0.001 \\
HDL (mmol/L) & $1.44(1.37-1.52)$ & $1.56(1.49-1.64)$ & 0.04 \\
LF/HF ratio & $1.55(1.20-2.01)$ & $1.02(0.79-1.31)$ & 0.03 \\
MBF rest $(\mathrm{ml} / \mathrm{min} / \mathrm{g})$ & $0.68(0.66-0.71)$ & $0.77(0.74-0.80)$ & $<0.001$ \\
MBF cold pressor $(\mathrm{ml} / \mathrm{min} / \mathrm{g})$ & $1.00(0.95-1.05)$ & $1.08(1.03-1.14)$ & 0.03 \\
MBF adenosine $(\mathrm{ml} / \mathrm{min} / \mathrm{g})$ & $2.35(2.25-2.45)$ & $2.32(2.22-2.43)$ & 0.73 \\
\hline
\end{tabular}

